Meta

Journal des traducteurs

Translators' Journal

\title{
La traduction en droit canonique
}

\section{Germain Lesage}

Volume 24, numéro 1, mars 1979

La traduction juridique

URI : https://id.erudit.org/iderudit/003478ar

DOI : https://doi.org/10.7202/003478ar

Aller au sommaire du numéro

Éditeur(s)

Les Presses de l'Université de Montréal

ISSN

0026-0452 (imprimé)

1492-1421 (numérique)

Découvrir la revue

Citer cet article

Lesage, G. (1979). La traduction en droit canonique. Meta, 24(1), 146-158.

https://doi.org/10.7202/003478ar d'utilisation que vous pouvez consulter en ligne.

https://apropos.erudit.org/fr/usagers/politique-dutilisation/ 


\section{La traduction en droit canonique}

Traiter aujourd'hui du droit canonique, c'est s'aventurer au sein d'une évolution qui, depuis un quart de siècle, en aura modifié profondément la nature.

Les lois de l'Eglise ont toujours revêtu un caractère juridique très particulier. Édictées depuis le $\mathrm{XII}^{\mathrm{e}}$ siècle selon les techniques du droit romain, elles ont en même temps comporté un esprit d'équité, ou un sens pastoral, qui leur conférait un caractère distinctif. Ainsi, dans le Codex juris canonici de 1917, on utilise plus de 1200 fois la conjonction «nisi - à moins que » ouvrant la porte à des exceptions. Les théoriciens du droit profane, droit continental ou Common Law, particulièrement les civilistes italiens, ont fortement dénoncé cet accroc majeur à l' « obligatorietà » juridique.

Mais voici que des événements récents : laïcisation de plus en plus universelle des États, insistance conciliaire sur la mission évangélique de l'Église entière, enracinement de la pensée ecclésiale dans les sources originelles de la foi, encouragent chez les théologiens et les canonistes une inspiration plus ecclésiologique.

Ce renouvellement de la doctrine entraîne aussi un renouveau des catégories, des concepts et du vocabulaire canoniques. En même temps, une désaffection quasi universelle pour la langue latine exige un usage généralisé des langues courantes dans les affaires de l'Église.

Terminologie nouvelle, audience différente, voilà qui s'ajoute à la spécificité même de la législation ecclésiale pour donner à sa traduction, dans le présent plus encore que dans le passé, un caractère distinctif. Nous nous proposons, dans le présent article, d'étudier tout d'abord l'évolution historique de la traduction canonique avant d'envisager la pratique actuelle.

\section{COUP D'CEIL HISTORIQUE}

L'universalité géographique de l'Église, sa centralisation hiérarchique et sa cohésion doctrinale exigent des normes communes mises à la portée de tous ses membres. Ceci implique nécessairement la traduction massive de ses lois. Pour saisir avec quelque justesse ce phénomène il faut examiner : 1) les langues canoniques et 2) la traduction canonique. 


\section{A. Les langues canoniques}

L'Église a toujours eu une langue commune, utilisée dans la liturgie, dans l'enseignement et dans la législation. Il s'agit d'un idiome en quelque sorte spécialisé, toujours vivant et en évolution, mais réservé aux clercs et aux penseurs. Dans la pratique religieuse populaire, le langage officiel était parfois transposé ou traduit dans les idiomes courants. Mais il reste que trois périodes se succèdent dans l'évolution linguistique du droit ecclésial : la période gréco-latine, des débuts au $\mathrm{IX}^{\mathrm{e}}$ siècle; la période latine, du $\mathrm{X}^{e}$ au $\mathrm{XVIr}^{\mathrm{e}}$ siècle ; la période \& polyglotte», du Xvar' siècle à l'époque actuelle.

\section{1) La période gréco-latine}

Du vivant même des apôtres, l'araméen, premiers idiome du christianisme, est supplanté par les deux langues classiques du temps : le latin et le grec.

Deux centres de pensée se développent, à Alexandrie d'Égypte et à Rome, exprimant chacun pour sa part l'esprit grec et l'esprit latin. La littérature ecclésiastique des premiers siècles consiste principalement en écrits théologiques, mais quelques cuvres portent sur l'organisation ou la structure institutionnelle de l'Église et prennent ainsi un caractère quasi canonique.

En Orient, on produit, au cours des $\Pi^{e}$ et $\mathrm{II}^{\mathrm{e}}$ siècles, des écrits apocryphes grecs qui tendent à exprimer les directives des douze apôtres. Bientôt, des synodes ou conciles particuliers groupent les évêques d'une même région, et leurs délibérations sont consignées en des textes conservés dans les archives diocésaines. En 325, le premier concile œcuménique, tenu à Nicée, dans la Turquie actuelle, démontre l'importance des Églises d'Orient. En 330, Constantin transfère le siège impérial de Rome à Byzance, qui devient alors Constantinople, aujourd'hui Istanboul. La langue courante et souvent officielle y est le grec.

En même temps, dès le début du me siècle, le secteur latin du christianisme se développe lui aussi. Comme leurs collègues orientaux, les évêques d'Occident, notamment ceux d'Afrique et des Gaules, tiennent des synodes fréquents dont les actes sont rédigés en latin et conservés aussi dans les archives locales. Au Ive siècle, principalement à la suite du départ de l'empereur, la papauté prend à Rome une importance prépondérante, tant au point de vue civil qu'au point de vue ecclésiastique. Les papes Damase (366-384) et Sirice (384-399) inaugurent la série des \& décrétales », ou lettres pontificales, qui constitueront subséquemment la source majeure du droit canonique, et qui toutes sont écrites en latin, car à Rome on ne sait plus le grec, et les rapports avec l'Orient y deviennent de pius en plus tendus et rares. Avec saint Léon le Grand (440-460), le caractère romaniste et latin de l'Église s'accentue et devient définitif.

Plus tard, d'autres conciles œcuméniques se tiendront en Orient, jusqu'à celui de 870 réuni à Constantinople. Mais déjà, la papauté s'est détournée de l'ancien Empire romain et s'est rapprochée du Saint Empire germanique. Le désaccord entre les secteurs occidental et oriental du christianisme devient officiel en 1054, avec le schisme de Michel Cérulaire. L'Église catholique est désormais latinisée. 


\section{2) La période latine}

Aux alentours du $x^{e}$ siècle, le latin est le médium universel de communication dans l'Église de Rome. Le peuple parle sans doute ses dialectes propres, mais tous les textes officiels sont rédigés dans la langue latine qui est celle des curies ecclésiastiques et civiles, des tribunaux, des universités, des publications, bref de tous les «clercs $\gg$ ou gens instruits.

L'usage du latin comme mode général d'expression est assuré par divers facteurs dont voici les plus notoines :

a) l'affermissement de l'autorité pontificale sous l'égide de grands papes canonistes tels qu'Alexandre III, Innocent III et Innocent IV ;

b) la fondation des universités, notamment celle de Bologne, où le droit romain et le droit canonique fleurissent de concert, et où enseignent des juristes et des canonistes éminents ;

c) la redécouverte du droit romain qui fournit aux législateurs et aux penseurs ecclésiastiques une structure, des institutions, des concepts, une terminologie ;

d) la prédominance du droit canonique non seulement sur les disciplines théologiques, mais aussi sur les divers droits séculiers, spécialement dans le domaine judiciaire, où l'Église apporte un personnel qualifié, un sens de l'ordre et de la justice que les Etats européens en formation ne peuvent encore établir ;

e) les collections de textes ecclésiastiques qui mettent le droit canonique à la portéc des autorités et des intellectuels; surtout celle du moine Gratien, publiée vers 1130 , sous le titre de Concordia discordantium canonum;

f) la vitalité des milieux universitaires bolonais, parisien, oxonien, etc., qui favorisent la production d'ouvrages, souvent monumentaux, « in utroque jure »;

g) la formation du Corpus juris canonici qui se poursuit du XIr au XvI siècle et constitue un instrument de pensée et d'action comparable au Corpus juris romain. Toute la législation ecclésiastique est ainsi réunie en une collection considérable, difficile d'accès sans doute, mais quand même réservoir massif de législation et de doctrine ;

h) le Concile de Trente (1545-1563), la réforme catholique ultérieure, l'établissement des Congrégations romaines, permettent à l'Église de s'adapter aux circonstances historiques et de s'engager dans une œuvre missionnaire universelle sous l'impulsion des papes et de la curie romaine.

Cet ensemble de circonstances favorise et utilise à plein l'instrument commun et adapté de communication qu'est la langue latine; non plus certes le latin classique des rhéteurs antiques, mais le latin scolastique, à la syntaxe plus simple et à la terminologie moins sélecte. 


\section{3) La période epolyglotte s}

Peu à peu, au cours de la Renaissance, le développement des langues modernes pénètre aussi le domaine canonique. Les textes officiels sont encore rédigés en latin, mais à Paris par exemple, des professeurs enseignent et écrivent en français. Au début du xvIII ${ }^{e}$ siècle fleurit en France une littérature où le droit canonique, le droit romain et le droit français s'entrecroisent.

$\mathrm{Au} \mathrm{XIX}^{e}$ siècle, l'accession des langues usuelles au niveau de la réflexion et de la recherche universitaires enlève au latin sa quasi-exclusivité comme moyen d'expression et de communication savantes. Dans le domaine canonique, deux faits nouveaux surgissent : D'une part, la publication de revues et de manuels didactiques qui mettent à la portée d'un plus grand nombre la législation et la science canoniques. Il ne s'agit pas de traduction officielles des textes légaux, mais de sommaires, de commentaires, de synthèses. D'autre part, la prolifération des séminaires pour la formation du clergé ainsi que le progrès des sciences ecclésiastiques accroissent le nombre des canonistes, professeurs et étudiants. En langue française, les professeurs de l'Université de Paris publient des ouvrages d'histoire, de jurisprudence, d'exégèse. En langue allemande apparaît la revue célèbre, encore existante «Archiv für katholischen Kirchenrecht.

$\mathrm{Au}$ tournant du $\mathrm{xx}$ siècle, à côté des ouvrages catholiques rédigés en latin, et qui restent les plus nombreux, on trouve une multitude de traités pratiques sur les institutions ecclésiastiques en général, sur la vie religieuse, etc., qui sont diffusés en langue courante.

\section{B. La traduction canonique}

Dans l'antiquité chrétienne, les rites orientaux possèdent un fond juridique de langue grecque dont les rites latins eux-mêmes ne peuvent se passer. L'inverse est également vrai. Ainsi, des traductions de textes grecs en latin et de textes latins en grec s'avèrent indispensables.

Au Moyen Âge, les chrétiens orientaux se séparent du catholicisme ou, à l'intérieur de celui-ci, forment des entités différentes entre elles et presque autonomes par rapport au secteur latin de l'Église avec lequel ils n'ont durant des siècles, que peu de rapports. Quant aux chrétiens occidentaux, leur très forte prédominance latine les éloigne des sources canoniques de langue grecque.

La Renaissance voit se développer une situation linguistique très différente, avec la formation des nations européennes. Le droit, en tant que législation et en tant que savoir, demeure l'apanage de spécialistes et s'exprime uniquement en latin. Il faut cependant appliquer le droit à la vie concrète de fidèles qui ne comprennent plus le latin. Mais plutôt que des traductions intégrales de textes canoniques, innombrables et diffus, on produit pour l'usage pastoral des synthèses simples et commodes en langue courante.

Depuis les débuts de l'Église jusqu'au $\mathrm{xx}^{\mathrm{e}}$ siècle, la traduction canonique n'a donc pas un rôle majeur. Toutefois, elle existe : 1) dans la législation ; 2) dans les sources et 3 ) dans la science canoniques. 


\section{1) La législation}

Dès que les circonstances amenèrent la papauté à remplir de plus en plus fréquemment le rôle dévolu au successeur de Pierre, un centre administratif se constitua à Rome. Des notaires, des archives, une chancellerie, un secrétariat étaient indispensables. Les requêtes et les appels venant de l'Orient comme de l'Occident, il fallait auprès du pape une équipe de traducteurs.

L'Église ne créa point à l'aveuglette un organisme imposant. Elle n'avait qu'à imiter, selon ses besoins et ses possibilités, la curie impériale où il fallait plus encore que dans l'Eglise satisfaire aux exigences administratives des deux secteurs, latin et grec. On peut même croire que les papes recouraient aux services des traducteurs impériaux, tout comme ils utilisaient à l'occasion les courriers publics.

On connaît peu de témoignages explicites sur la traduction en grec de documents latins émanant des papes ou des conciles. Mais il s'en trouve un qui est tout à fait clair. Il s'agit d'un message où saint Léon le Grand demande à l'empereur Marcien de faire traduire en grec et de diffuser une lettre qu'il a écrite à Flavien au sujet du nestorianisme : « ... je prie votre vénérable clémence d'ordonner que cette même lettre, traduite en langue grecque, intégralement et diligemment, par mon confrère l'évêque Julien ou par ceux que votre piété aura choisis comme aptes à ce travail, soit transmise par un porteur sûr et sous la marque de votre sceau... ${ }^{1}$,

\section{2) Les sources}

L'entreprise de traduction la plus importante peut-être de toute l'histoire du droit canonique est celle de Denys le Petit. Vraisemblablement sous le règne du Pape Gélase (492-496), ce moine scythe, qui devait décéder vers 550, traduit en latin tous les documents canoniques de langue grecque alors connus à Rome.

Un essai modeste avait paru auparavant ; mais Denys annonce qu'il se met à l'œuvre à son tour pour pallier les insuffisances de cette version déjà existantes : «... imperitia... priscæe translationis ${ }^{2} »$.

Connue sous le titre de Dionysiana, cette nouvelle traduction constitue la plus importante des sources antiques du droit de l'Église. Elle a servi de base à toutes les grandes collections subséquentes; le nombre considérable des textes qu'elle contient, et dont la plupart sont authentiques, ainsi que la multitude des thèmes traités en font une somme pratiquement complète de la discipline ecclésiastique, telle qu'elle existe vers la fin du ve siècle, d'après les canons conciliaires et les lettres pontificales.

\section{3) La science}

Depuis la Renaissance, le savoir canonique s'exprime de plus en plus en langue courante. Les auteurs doivent donc traduire pour leur clientèle le texte

1. Léon le Grand, Ad Marcianum Augustum, Epistola CXXX, Cap. III, in Patrologia latina, t. 54, col. 1081.

2. Die Canonessamlung des Dionysius Exiguus in der ersten Redaktion, Hrsg. von Lic. Adolf Strewe, Berlin und Leipzig, W. de Gruyter, 1931, p. 1. 
latin des lois. Les failles existant chez certains d'entre eux illustrent les difficultés qu'ils affrontent.

Ainsi, un médiéviste contemporain cite comme suit un message du Pape Alexandre III au Roi Henri II d'Angleterre : « Tu ne crains pas, non seulement de prendre, comme c'est convenable, ce qui est à César, mais aussi d'usurper au péril de ton âme ce qui est à Dieu - Non solum quæ sunt Cæsaris, prout convenit, obtinere, sed et quæ Dei sunt illicite atque in animæ tuæ periculum usurpare, non metuis. "L'omission du mot \&illicite» n'est certes pas une faute légère ${ }^{8}$.

Une mention spéciale est due à la traduction des Regula juris canonici publiée en 1720 par Jean-Baptiste D'Antoine. Quelques spécimens permettent de l'évaluer.

« Regula VI. Textus. Nemo potest ad impossibile obligari. Version. Nu1 ne peut être obligé à l'impossible.» "Regula XIX. Textus. Non est sine culpa, qui rei, quæ ad eum non pertinet, se immiscet. Version. C'est une imprudence, \& souvent une témérité de se mêler des affaires d'autrui, où l'on n'a aucune part, \& l'on devient par son fait responsable des événements. " Regula XXIX. Textus. Quod omnes tangit, debet ab omnibus approbari. Version. Le consentement de tous les intéressés est requis dans une affaire commune entre plusieurs personnes.» \& Regula XLVIII. Textus. Locupletari non debet aliquis cum alterius injuria, vel jactura. Version. L'on ne doit pratiquer aucune injustice pour s'enrichir de la perte d'autrui ${ }^{4}$.

Ces extraits, qui vont de la version littérale à la paraphrase, montrent l'absence d'une théorie consistante de la traduction chez D'Antoine, semblable en cela à beaucoup d'autres. Tout au plus, les auteurs essaient-ils d'appliquer le principe global énoncé par saint Thomas d'Aquin : «Il incombe à un bon traducteur qu'en traduisant ce qui concerne Ia foi catholique, il conserve le sens en changeant la façon de parler d'après le caractère propre de la langue dans laquelle il traduit ${ }^{5}$.

\section{II, SITUATION ACTUELLE}

$\mathrm{Au}$ cours de l'histoire, la diversité des langues n'a pas affecté profondément le droit canonique. Depuis le vre siècle, et surtout le $\mathrm{Xr}^{\mathrm{e}}$, jusqu'au code de 1917 , le latin sert de véhicule commun à la pensée canonique. Toutes les personnes immédiatement intéressées au droit de l'Église comprennent le latin; les ouvrages techniques sont publiés en latin; c'est en latin aussi que les études sont poursuivies et que les échanges de vues sont effectués.

3. Marcel Pacaut, Alexandre III. Etude sur la conception du pouvoir pontifical dans sa pensée et dans son cuvre, Paris, Vrin, 1956, p. 165. Voir : Germain Lesage, "L'Autorité ecclésiale d'après Alexandre III *, dans Studia Canonica, vol. 7, 1973, p. 33.

4. Jean-Baptiste D'Antoine, les Règles du droit canon, nouvelle éd, Liège, J. Dessain, 1773, p. $47,116,181,264$.

5. Thomas d'Áquin, Contra errores Graecorum ad Urbanum IV, P.M., dans Sancti Thomae Aquinatis Opera omnia ad fidem optimarium editionum accurate recognita, Parmae, Tipis.P. Fiaccadori, tomus XV, 1864, p. 239 . 
Mais il en va tout autrement aujourd'hui, de sorte que pour comprendre la pratique actuelle de la traduction canonique, il faut percevoir certains éléments groupés ici sous trois titres : 1) renouvellement du système canonique ; 2) importance accrue de la traduction ; 3) difficultés propres au domaine.

\section{A. Renouvellement du système canonique}

Le caractère spécifique du droit de l'Église s'est modifié profondément depuis le début du siècle, par suite de trois événements principaux : la promul-

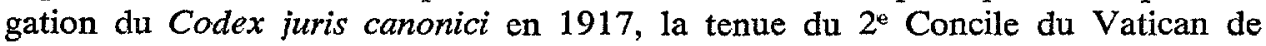
1962 à 1965, le projet de révision du Codex, en marche depuis 1963.

\section{1) Le Codex juris canonici}

Le code de 1917 a été publié officiellement dans la seule langue latine. Vraisemblablement, il n'a jamais existé de traduction complète si ce n'est en espagnol.

De fait, une traduction complète ne s'imposait pas, puisque les clercs, qui seuls étaient vraiment impliqués dans le droit canonique, savaient tous assez bien le latin. Les normes utilisées dans le ministère pastoral étaient exposées d'une façon synthétique dans des traités plus accessibles, publiés en langues courantes.

Après la publication du code, l'accroissement de l'Église dans les pays anglophones posa cependant un problème de plus en plus sérieux par suite du peu de maîtrise de la langue latine au sein de leur clergé local. Mais en même temps que les nécessités pastorales grandissaient, la publication en anglais d'ouvrages canoniques de type pratique se développait également.

\section{2) Le concile de 1962-1965}

Le $2^{\text {e }}$ Concile du Vatican est marqué par une orientation doctrinale dont les conséquences en droit canonique sont considérables.

Rompant avec une tradition quasi millénaire, une école ecclésiologique dont le coryphée est le père Yves Congar, o.p., prône un retour aux visions spirituelles des origines chrétiennes. Sans que les concepts fondamentaux soient modifiés, des insistances apparaissent en divers aspects. Ainsi, c'est le peuple de Dieu, pris dans son ensemble, que l'on met au premier plan dans l'Église, de préférence à l'autorité hiérarchique des pasteurs.

Il en résulte, dans le domaine du droit canonique, de multiples modifications destinées à introduire une nouvelle inspiration, de nouveaux concepts, une nouvelle terminologie.

Le droit ecclésial empruntera désormais ses techniques de base, ses orientations d'ensemble, son esprit, ses valeurs institutionnelles, ses structures concrètes, non plus tant au droit romain qu'à la «sagesse 》 théologique. Ce sens ecclésiologique introduit un renouveau de nombreuses institutions, qui devien- 
nent moins juridiques et plus spirituelles, et une rénovation de la terminologie qui remet en faveur des expressions paléochrétiennes telles que koinonia, diaconia, etc.

\section{3) La révision du Codex}

Après quinze ans de travaux, le projet de révision du code de droit canonique, mis en ceuvre par le Pape Jean XXIII, vient d'atteindre sa phase finale.

Les sept livres du futur code, préparés par des commissions spéciales et revus par le Pape Paul VI, ont été distribués aux évêques et aux universitaires de toute l'Église, dans un processus de consultation préalable à la rédaction définitive et à la promulgation.

Les textes du projet - ou schemata - sont rédigés en latin, de même que les explications proposées, au fur et à mesure du déroulement des travaux, dans une revue ad hoc intitulée Communicationes.

Le texte projeté est "réservé», en ce sens que sans être soumis au secret officiel et absolu, il devrait être réservé à l'usage des prélats, canonistes ou spécialistes impliqués. Le motif de cette discrétion est d'éviter des incompréhensions ou des critiques stériles à ce stade, encore provisoire et imparfait, d'une œuvre fort complexe.

Toutefois, en raison de l'importance de certains textes, ou de l'âpreté des discussions sur certains thèmes, plusieurs des projets ont quand même été traduits et diffusés en italien, anglais et français. Ces indiscrétions auront permis d'apporter des corrections et des améliorations aux ébauches.

\section{B. Importance accrue de la traduction}

Quoique l'usage de la langue italienne s'accroisse dans les communications ordinaires de la curie romaine, c'est encore le latin qui demeure l'unique langue officielle des textes canoniques d'ordre public.

Chaque document proprement juridique émanant des dicastères romains doit donc être traduit en une multitude de langues.

Les principaux aspects de cette entreprise sont les suivants : 1) son rôle ; 2) ses genres ; 3) ses difficultés ; 4) le personnel qui s'y consacre et, 5) la technique utilisée.

\section{1) Rôle}

Le but propre du droit canonique est de concrétiser et de garantir, sur le plan extérieur et social, par le truchement d'institutions spécifiques, la finalité évangélique de l'Église.

Cette ordonnance de l'activité ecclésiale procède des autorités universelles à Rome et des autorités locales dans les diocèses.. Ces dernières, bien sûr, rédigent leurs actes dans les idiomes de leurs contrées respectives, mais les dicastères 
romains s'en tiennent généralement au latin comme véhicule officiel de leurs directives d'ordre public.

Les traductions de ces textes pontificaux doivent donc viser à assurer, parmi les croyants de tout l'univers, l'authenticité de la foi, l'unité des rites et de l'apostolat, l'efficacité de la communication.

\section{2) Genres}

On trouve communément, dans le champ du droit canonique, trois sortes de traduction.

La première est la traduction de textes latins en des langues usuelles. C'est la moins difficile des trois.

La deuxième est la version, en une langue courante, de documents originellement rédigés aussi en une langue vivante. Cette traduction n'est pas sans embûches, surtout s'il s'agit soit de termes techniques, soit de termes populaires. Ainsi, l'expression «débile psychopathe », qui signifie une personne rendue débile par un mal psychique, peut être comprise, par un Italien, comme désignant une personne faiblement psychopathe; ce qui, en jurisprudence matrimoniale, par exemple, n'est pas sans conséquence. Un autre fait, tiré d'une cause de canonisation, illustre une difficulté analogue provenant d'une expression populaire. Il s'agissait de la guérison d'un aveugle, admissible comme miracle seulement si le bénéficiaire, d'abord totalement aveugle, avait subitement recouvré la vue d'une façon parfaite. Un témoin canadien affirme que l'aveugle en question \& ne voyait pas clair» avant l'invocation du serviteur de Dieu, mais que, par la suite, il «voyait clair». Il fallut expliquer à l'équipe romaine de spécialistes que pour un Canadien, \& voir clair \& signifie simplement voir! Donc, le miraculé, qui d'abord ne voyait pas du tout, voyait très bien par la suite.

La troisième espèce de traduction, la plus difficile, consiste à transposer en droit canonique et à rendre en latin des concepts tirés de systèmes juridiques profanes.

\section{3) Difficultés}

On peut ramener succinctement les difficultés ordinaires de la traduction canonique aux quatre causes que voici :

a) le caractère spécialisé, quasi ésotérique du droit ecclésial, qui traite de thèmes souvent étrangers aux réalités profanes;

b) la pluralité culturelle des croyants, qui exige une multitude de versions, une masse énorme de textes à traduire et un nombre effarant de traducteurs ;

c) les paramètres provenant de la révélation et qui exigent une exactitude irréprochable de la traduction. Saint Thomas d'Aquin, dans le passage cité plus haut, excuse les erreurs de certains théologiens par le fait qu'ils devaient se servir de traductions fautives ; 
d) le projet spécifique du christianisme, c'est-à-dire la perfection personnelle des croyants, visée « maximale » qui dépasse une rigidité strictement juridique, visant simplement à un ordre public minimal consistant dans la prévention ou la correction des crimes majeurs contraires à la liberté ou aux droits des citoyens.

\section{4) Personnel}

Le travail de traduction canonique ne s'exécute généralement pas, dans l'Église, par un bureau central de la Curie romaine. Il procède plutôt d'organismes particuliers et de traducteurs individuels.

Les principaux organismes qui veillent à la traduction des documents romains sont les conférences épiscopales. Ainsi, le Directoire du ministère pastoral des évêques a été traduit en français et en anglais par les soins de la Conférence des évêques catholiques du Canada. D'une façon régulière, certaines revues présentent des versions de documents canoniques importants ; telles sont la Documentation catholique de Paris et Origins de Washington. Une collection de versions anglaises, Canon Law Digest, a déjà publié aux États-Unis sept volumes imposants. Actuellement, des sociétés de droit canonique, celles des États-Unis notamment, s'occupent de la traduction des différents schemata qui constitueront le prochain code.

Les traducteurs individuels sont de plus en plus rares. La déchéance du latin comme langue d'Église, ou comme langue des clercs, ainsi que la réduction des études canoniques chez les candidats aux ordres fait que seuls de rares spécialistes, professeurs d'université ou officiers de curie, connaissent suffisamment et le droit ecclésial et la langue latine pour réussir de bonnes traductions.

\section{5) Technique}

On ne saurait parler d'une théorie ou d'une technique de la traduction canonique comme telle. On trouve à Rome des équipes de latinistes qui polissent les textes officiels, ou des traducteurs professionnels affectés au Tribunal de la Rote. Ailleurs, des organismes embauchent des traducteurs compétents, mais on n'y découvre guère un véritable système.

Il semble que les traducteurs qui sont aussi canonistes visent plutôt à une version littérale, tandis que ceux qui sont davantage théologiens ou lettrés préfèrent une cuvre littéraire. En pratique, on rencontre tous les types de versions, depuis la plus strictement littérale jusqu'à la paraphrase, comme il appert de l'ouvrage de D'Antoine cité précédemment.

\section{Difficultés propres au domaine}

Quelques exemples peuvent illustrer les difficultés typiques de la traduction canonique, notamment celles qui surgissent : 1) de l'introduction d'un nouveau vocable; 2 ) de la rédaction d'une norme à teneur évangélique et, 3) de l'emprunt, à des systèmes profanes, d'une institution juridique qui leur est propre. 


\section{1) Un vocable nouveau}

Désireux de recourir à un terme plus général ou moins équivoque que celui de «supérieur» pour désigner le détenteur de l'autorité dans les instituts de vie consacrée, le schema préparatoire du futur droit utilise le mot moderator.

Ce vocable a suscité beaucoup d'opposition et illustre bien une situation qui se produira maintes fois dans le nouveau code. Il s'agit en effet d'un mot impeccable au point de vue doctrinal ou linguistique, mais ambigu dans son usage populaire.

Dans le sens courant, le terme français \& modérateur » connote l'idée de modération, de refrènement, d'opposition au dynamisme, à la liberté. Toutefois, au point de vue sémantique, la signification de moderator est positive ou constructive. En latin, le moderator «... est qui moderatur, gubernator, rector ${ }^{6}$ ». En français, modérateur ou modératrice signifie « ... celui, celle qui modère, qui dirige, qui règle ${ }^{7} \gg$. En anglais, le sens est identique : un moderator est $\&$... a ruler, governor, director ${ }^{8} \gg$.

Il serait donc loisible de traduire en français moderator par modérateur, mais il faudrait escompter que l'usage canonique finirait par prévaloir sur l'usage populaire.

\section{2) Une norme évangélique}

Le même schema sur la vie consacrée contient une norme d'inspiration évangélique sur le caractère essentiel de la vie religieuse qui est la « sequela Christi ». Non seulement le mot sequela est-il lui-même pratiquement intraduisible, mais c'est aussi la norme où on l'exprime qui est difficile à traduire à cause, pour une bonne part, de son caractère plus théologique que juridique. Examinons le texte original et trois versions de cet article du schema.

a) Texte latin : «Permulta in Ecclesia sunt instituta vitæ consecratæ variis Spiritus charismatibus ornata, quæ donationes habent, secundum gratiam quæ data est eis, differentes : Christum enim pressius sequuntur vel orantem, vel actuosa operositate hominibus benefacientem, vel cum eis in sæculo conversantem. »

b) Version française européenne : "Très nombreux dans l'Église sont les instituts de vie consacrée ornés de charismes divers reçus de l'Esprit, qui ont des dons différents selon la grâce qui leur a été accordée : en effet, ils suivent le Christ de plus près, dans la prière, le service des hommes ou la vie avec eux en plein monde.

c) Version française canadienne : Il y a dans l'Église de nombreux instituts de vie consacrée, ornés de charismes variés de l'Esprit, qui ont des dons

6. Egidius Forcellini, Lexicon totius latinitatis, Patavii, Typis Seminarii, 1940, t. 3, p. 265.

7. Emile Littré, Dictionnaire de la langue française, édit. intégrale, Paris, Pauvert, 19561958 , tome 5, p. 312. 8. William Little et al., The Shorter Oxford English Dictionary on Historical Principles,
3 rd ed., Oxford, The Clarendon Press, 1965, p. 1268 . 
différents selon la grâce qui leur a été donnée : en effet, ils suivent de plus près le Christ, soit priant, soit faisant du bien aux hommes par son labeur actif; soit vivant avec eux dans le siècle.

d) Version anglaise (Etats-Unis) : $<$ There are in the Church very many institutes of the consecrated life, endowed with varied charisms of the Holy Spirit and possessing different gifts according to the grace given them. They more closely, follow Christ praying, or Christ performing good deeds for people through active works, or living among people in the world. >

On remarque donc que la première version française comporte une double ambiguïté dans les dernières lignes; que la seconde est très littérale; que la version anglaise frôle la paraphrase en voulant éviter l'ambiguïté commise par le premier traducteur français.

\section{3) Des institutions « empruntées *}

Le renouveau conciliaire introduit en ecclésiologie et en droit plusieurs termes grecs qui désignent des institutions primitives. Certains de ces mots sont pratiquement impossibles à traduire avec fidélité. Comment, par exemple, rendre le sens adéquat de koinonia? Le terme * communion se présente naturellement à l'esprit, mais on peut douter qu'il traduise exactement tout le contenu historique et doctrinal du grec originel.

De son côté, le droit romain prête lui aussi au droit canonique des institutions et un vocabulaire. Il est généralement possible d'en découvrir la signification dans les ouvrages qui traitent du système juridique de Rome. Mais ce n'est pas toujours facile. Comment, par exemple, traduire en français l'expression consortium ou communio insérée dans la définition canonique du mariage ? Le mot « communion » se présente ici encore, mais est-il vraiment juste ? Fréquemment, après avoir traduit d'une façon aussi équivalente que possible le terme latin, il vaudrait mieux, ce semble, le répéter dans la version. Voici un exemple pris dans la décrétale « Sæpe contigit \& de Clément $\mathrm{V}$ : Citationem vero ac prestationem juramenti de calumnia vel malitia, sive de veritate dicenda, ne veritas occultetur, per commissionem hujusmodi intelligimus non excludi. \$ Traduisons : «Par un tel mandat, nous entendons ne pas exclure la citation ou la prestation du serment de droiture - de calumnia ou de bonne foi - malitia et de sincérité - de veritate dicenda, afin que la vérité ne soit pas cachée ${ }^{\circ}$.

Enfin, signalons que l'utilisation de vocables tirés des systèmes profanes contemporains ne va pas non plus sans complications. Ainsi, doit-on traduire le mot italien ordinamento ou le mot espagnol ordenamiento par le correspondant littéral : ordonnance, ou par le synonyme : système? Quant à l'expression espagnole acto negocial provenant de la philosophie du droit, pourrait-elle signifier en français l'acte d'implication communautaire ${ }^{10}$ ? Dans la même veine, ne

9. Aemilius Friedberg, Corpus juris canonici, tomus 2, Lipsiae, Tauchnitz, 1881, col. 1143 ; aussi Germain Lesage, o.m.i., \& Procédures matrimoniales d'après le Schema De Pro cessibus 》, dans Studia Canonica, vol. 11, 1977, p. 215.

10. Voir : Germain Lesage, o.m.i., L'Autonomie privée dans le droit de l'Eglise », dans Jus canonicum, vol. 15, 1975, p. 188. 
faudrait-il pas renoncer à traduire le terme due process créé par le Common Law étatsunien?

La traduction canonique est sans doute peu passionnante ; mais elle exige tout de même une grande science, une grande culture et un grand art.

Rien d'étonnant donc qu'on y décèle des vicissitudes.

Il est certes heureux, face à tant d'impasses, que le droit canonique ne soit pas toute l'Église, ni même ce qu'il y a de plus important dans la vie concrète de l'Église, mais qu'il soit simplement un outil » au service du mystère d'amour qui, lui, est l'Église.

Germain Lesage 